\title{
VISIBILIDADE E DIREITO: ESBOÇO DE UM PROBLEMA
}

\section{Eduardo Ramalho Rabenhorst}

Professor Titular da Universidade Federal da Paraíba (UFPB)._Email: rabenhorst_l@hotmail.com.

Resumo: O propósito deste artigo é apresentar um esboço de investigação sobre a relação entre cultura visual (visual culture) e direito. Ainda que incipientes, os estudos sobre o tema estimulam uma reflexão teórica sobre o reconhecimento social e jurídico de grupos minoritários ou vulneráveis, e oferecem novos pontos de vista sobre os temas da segurança e da representação política em uma era marcada pelo predomínio da visibilidade.

Palavras-chave: Visibilidade; cultura visual; direitos; segurança; democracia.

Abstract: This paper presents an outline of research on the relationship between visual culture and law. Although incipient, studies on the subject stimulate a theoretical reflection on the social and legal recognition of

\footnotetext{
${ }^{1}$ Conforme observa Nathalie Heinich, o termo visibilidade denota no âmbito das ciências sociais simultaneamente as ideias de reconhecimento, publicidade e "observalidade", o que torna difícil sua conceitualização. Cf. HEINICH, Nathalie. De la visibilité. Excellence et singularité em régime médiatique. Paris: Gallimard, 2012. Andrea Brighenti, por sua vez, estabelece uma distinção entre três sentidos do
}

minority or vulnerable groups, and offer new points of view on the themes of security and political representation in an era marked by the predominance of visibility.

Keywords: visibility, visual culture, rights, safety, democracy.

\section{Introdução}

Visibilidade é uma palavra com geometria variável, muito recorrente no debate acadêmico e nas artes contemporâneas ${ }^{1}$. Isso porque estamos a viver em uma época que se entregou à visibilidade. Com efeito, faz-se presente, em nossos dias, a coação da exposição, essa exigência permanente de visibilidade, de ver e de se fazer visível, sobretudo através das plataformas digitais de difusão: web,

termo visibilidade: "visibilidade social", associada ao reconhecimento de direitos; "visibilidade mediática", associada à exposição e à difusão de imagens; e a visibilidade enquanto controle, ligada à vigilância. Cf. BRIGHENTI, Andrea. "Visibility. A Category for the Social Sciences", Current Sociology, Vol. LV, n 3, maio de 2007. 
redes sociais, blogs, etc. A injunção dessa da visibilidade se dá em concomitância com o surgimento de uma cultura da imagem, da transparência e das telas. A televisão, o computador, o smartphone e o GPS constituem alguns dos painéis eletrônicos aos quais os indivíduos contemporâneos dedicam o seu tempo de forma vertiginosa ${ }^{2}$.

Ora, em uma época em que as experiências visuais se tornaram tão centrais, constituindo-se em modo privilegiado de expressão e de comunicação, não é de causar espanto o surgimento de uma área de estudos interdisciplinar que vem recebendo o nome de "cultura visual". A expressão "cultura visual" talvez não seja muito feliz, já que cultura é um termo pretensioso, com um largo campo de expressão. Porém, o que se pretende ao se falar em "cultura visual" é enfatizar a cultura como um processo de

\footnotetext{
${ }^{2}$ Para uma história do surgimento da cultura da tela no Ocidente e dos processos de transformação da percepção e da atenção que resultaram na modificação radical do estatuto da imagem a partir do século XIX, veja-se CRARY, Jonathan. Techniques of Observer. On vision and modernity in the XIX century. Massachusetts, MIT Press, 1992.

${ }^{3}$ Como elucida Gonzalo Abril, a cultura visual "é uma forma de organização sócio-histórica da percepção visual, da regulação das funções da
}

produção e circulação de sentidos dentro de um grupo ou sociedade, algo que se dá por meio de práticas e discursos comuns. $O$ que se busca destacar com a expressão "cultura visual" é, portanto, o fato de que alguns aspectos da cultura são "eventos visuais": imagens, objetos e experiências. Em outros termos, que os diversos grupos humanos constroem, cultural e historicamente, maneiras diferentes de visualizar e de representar visualmente a realidade (modelos de visibilidade, agentes, processos e objetos) ${ }^{3}$.

Falar de "cultura visual" é questionar a ideia de universalidade da experiência visual. É reconhecer que a visualização não pode ser reduzida ao conjunto de operações ópticas, químicas e nervosas envolvidas na visão, mas que o "ver" é uma operação igualmente submetida a uma gestão social, isto é, que se trata de uma prática

visão e de seus usos epistêmicos, estéticos, políticos e morais". Do mesmo modo, é uma maneira socialmente organizada "de criar, distribuir e inscrever textos visuais, processo que implica sempre umas determinadas tecnologias de fazer visível, técnicas de produção, reprodução e de arquivo". Cf. ABRIL, Gonzalo. Cultura visual, de la semiótica a la política. Madrid: Plaza y Valdés, 2013, p. 35. 
afeita a um conjunto de instruções bastante precisas: quem vê, quem é visto; como se vê, como se é visto ${ }^{4}$ O que se busca destacar nessa ideia é, essencialmente, o fato de que há regulações do ver e do ser visto, dos modos de fazer ver e dos modos de ser visto. Tais regulações, chamadas de "regimes de visibilidade", são historicamente situadas, dependem de uma série de instituições e estão submetidas à lógica do poder político, ou seja, cada sociedade decide, em uma situação dada, utilizando um meio específico, tornar algo visível ou invisível $^{5}$.

É necessário pois diferenciar a visualidade - que se refere ao dado físico que é percebido pela vista, ligada às capacidades físicas de nosso sentido visual e às propriedades espaciais e temporais das circunstâncias nas quais o/a visualizador/a se encontra (campo de visão) - da visibilidade propriamente dita, que diz respeito aos mecanismos socioculturais partilhados que constituem e regulam os modos de ver

${ }^{4}$ Cf. SAUVAGEOT, Anne. Vois et Savoirs. Esquisse d'une sociologie du regard ", PUF, 1994.

5 A noção de "regime de visibilidade" designando os modos como o "visível" e o "invisível" são construídos socialmente e
195

e as experiências visuais, isto é, às estratégias que transformam a percepção psicofísica em fluxo propriamente significativo.

$\mathrm{O}$ interesse pelo estudo da "cultura visual" seria limitado a algumas disciplinas específicas, especialmente a antropologia e a história da arte, não fosse o fato de que as experiências visuais se tornaram paradigma de nossa época. Vivemos em uma cultura que modificou completamente a concepção da percepção visual em termos de modo de produção do fenômeno visual e do surgimento de novos objetos de visão, uma cultura que fez massivamente da imagem o meio privilegiado de representação, produção de significado e de comunicação. Daí a ideia de alguns autores de que após o linguistic turn, que caracterizou o pensamento no século $\mathrm{XX}$, teria chegado agora o momento de se propor um visual turn, que atribuiria ao visual ou ao icônico o papel de tópico central das discussões ${ }^{6}$.

regulados atravessa a obra de Michel Foucault. Sobre a noção de régime de visibilidade cf. FISEROVÁ, Michaela. Partager le visible. Repenser Foucault. Paris: L'Harmattan, 2013. 6 Cf. MIRZOEFF, Nicholas. Visual Culture Reader. London: Routledge, 1998, p. 5. 
No âmbito dos estudos sobre cultura visual, interessa compreender, por exemplo, como o visual ganhou tamanha amplitude no mundo moderno em detrimento do invisível e do secreto, mas também em prejuízo da própria "palavra" como forma de expressão. Por outro lado, os estudos sobre cultura visual também buscam compreender as modificações impostas por essa centralidade da visão em diferentes níveis de funcionamento da sociedade (e.g., trabalho, lazer, conhecimento, etc.) e entender as transformações infligidas aos outros modos de ser e de sentir dos indivíduos, já apontadas por Walter Benjamin em seu estudo clássico sobre a obra de arte na época de sua reprodutibilidade técnica ${ }^{7}$. Outrossim, os estudos sobre cultura visual perquirem ainda como a visibilidade se tornou um valor central da política, afetando de modo particular a própria compreensão da democracia como exercício público do poder ${ }^{8}$.

Ora, em que medida é que as discussões sobre visão, visualidade e visibilidade interessariam ao direito?

7 Cf. BENJAMIN, Walter. L'CEuvre d'art à l'époque de sa reproductibilité technique. Paris, Payot: 2013.

${ }^{8}$ Cf. BRIGHENTI, Andrea. La démocratie a l'heure des visibilités hiérarchisées. In:
196

Em outros termos, haveria aspectos jurídicos do tema da visibilidade? Para além das tradicionais questões atinentes à imagem, que interessam os/as juristas desde o surgimento da fotografia e do cinema, e mais recentemente as questões específicas suscitadas pelo surgimento das imagens numéricas, parece evidente que há outros aspectos de natureza mais filosófica e sociológica que também deveriam suscitar interesse entre os/as juristas, sobretudo aqueles/as que se dedicam à reflexão teórica sobre o direito.

Com efeito, Andrea Brighenti (2009) tem procurado mostrar em seus escritos que a visibilidade faz confluir o ato físico de ver, as novas tecnologias do visual e os elementos discursivos que elaboram o que é a visão, ou seja, que a visibilidade conjuga relações de percepção (aspecto estético) com relações de poder (aspecto político). Para Brighenti, os efeitos da visibilidade dependem de arranjos territoriais, relacionais, organizacionais e tecnológicos particulares, o que evidencia o papel importante que

FABRE, Thierry. La cité en danger. Dictature, transparence et démocratie. Rencontres d'Averroès 19, Marseille: Éditions Parenthèses, 2013. 
desempenha o direito nesse processo enquanto modalidade de simbolização da ação humana por intermédio de normas. Neste processo de construção da visibilidade estão confluídos o ato físico de ver, as novas tecnologias do visual e os elementos discursivos que elaboram o que é a visão. A visibilidade conjuga, assim, relações perceptivas com relações políticas ${ }^{9}$.

Seguindo essas pistas de investigação estabelecidas pelo sociólogo italiano, tentar-se-á destacar nesse breve artigo três aspectos da visibilidade que podem interessar a uma reflexão teórica sobre o direito: (1) a visibilidade como forma de reconhecimento social, especialmente a visibilidade de grupos minoritários ${ }^{10}$; (2) a visibilidade enquanto vigilância e controle; e (3) a visibilidade mediática e a democracia representativa.

\section{Visibilidade e reconhecimento social}

9 Cf. BRIGHENTI, Andrea. Pour une territoriologie du droit, In: FOREST, Patrick (Dir.) Géographie du droit. Epistémologies, développements et perspectives. Québec: Presses de l'Université Laval, 2009, pp. 239260.

10 Categoria de análise do campo jurídico que indica, geralmente, grupos em situação de vulnerabilidade.
A capacidade de ver e de ser visto, como aqui já foi dito, não repousa apenas em qualidades naturais, mas é algo que depende largamente de regras sociais, podendo inclusive se transmutar em exigência política em torno da qual os atores individuais e coletivos entram em confronto ${ }^{11}$. A luta por visibilidade, conforme observou Axel Honneth, é parte da "luta por reconhecimento", ainda que "ser visível" não seja garantia de ser "reconhecido"12. No entender de Honneth, há dois momentos que configuram o ato de reconhecimento: o momento da identificação cognitiva, que ocorre quando o sujeito ou o grupo é visto pelo outro a partir de suas características particulares, e o momento da expressão, quando ocorre a demonstração pública deste reconhecimento ${ }^{13}$.

No âmbito de uma sociedade liberal, quando indivíduos ou grupos são invisíveis, seja de modo absoluto ou

11 Cf. VOIROL, Olivier. Visibilité et invisibilité: une introduction. Reseaux, $\mathrm{n}^{\circ} 129$ 130, 2005, p. 9-36.

12 Cf. HONNETH, A. «L'invisibilité: sur l'épistémologie de la reconnaissance». Réseaux, vol. 23, n. 129-130, p. 41-57, 2004. 13 Cf. HONNETH, A. La lutte pour la reconnaissance. Paris: Éditions le Cerf, 2005. 
de modo relativo, isso significa, do ponto de vista jurídico, que as estruturas legais criadas para promover a liberdade e a igualdade não funcionam ou que elas funcionam com menos eficácia e rigor quando se trata de determinados segmentos. A invisibilidade social é um caso típico. Ela remete à ausência de reconhecimento social de públicos excluídos dos espaços de representação e de participação cidadã, tal como acontece com as pessoas expostas à situação de rua, entre outras.

A invisibilidade social, além de denegar a participação justa dos grupos na cena pública, serve para reforçar ideias estereotipadas acerca de seus membros. Muitos dos movimentos em favor de direitos de um determinado grupo sociocultural são movimentos por visibilidade. Do mesmo modo, muitas violações de direitos humanos estão conectadas à questão da visibilidade: mulheres, pessoas com

\footnotetext{
14 Não é possível efetuar aqui uma discussão mais aprofundada da relação entre justiça e visibilidade. Seguindo Rawls pode-se dizer, entretanto, que injusta é toda situação que institui ou aumenta desigualdades entre os sujeitos e grupos sem melhorar ao máximo a situação daqueles que são menos favorecidos. Nos exemplos anteriormente apontados, o que está em jogo é uma desigual capacidade de
}

198

identidades de género e sexualidades não-normativas, pessoas com diversidade funcional, pessoas expostas à situação de rua, pessoas em contexto prisional, pessoas diagnosticadas com doença mental em situação de internamento, imigrantes, etc. Daí a pertinência de se falar, ao menos prima facie, de uma visibilidade justa ou injusta ${ }^{14}$.

Mas é preciso observar, no entanto, que a visibilidade não opera sem ensejar ambivalência, o que torna a discussão bastante complexa. Como mostraram N. Aubert e C. Haroche ${ }^{15}$, alguns grupos sociais, por exemplo, são invisíveis sob certo ângulo, mas eles são excessivamente visíveis sob outro prisma. $\mathrm{O}$ caso das mulheres é bastante ilustrativo: invisíveis do ponto de vista político, por exemplo, elas são, em contrapartida, excessivamente "visíveis" nos meios de comunicação social e na publicidade, aparecendo sobretudo a partir de representações

apropriação do espaço e de imposição de seu uso por parte de determinados grupos, revelando assimetria que deveria ser regulada pelo poder público. Cf. RAWLS, John. A Theory of justice. Harvard Universty Press, 1999.

15 AUBERT, Nicole e HAROUCHE, Claudine. Tyrannies de la visibilité. Être visible pour exister? Paris: ERES, 2011. 
visuais que reforçam estereótipos de gênero.

O mesmo pode ser dito em relação a outras manifestações da visibilidade operadas pelos meios de comunicação social que manipulam códigos de representação e expõem a vida íntima e os laços afetivos na vida pública: reality shows, revistas de celebridades, redes sociais, etc. Para além das questões de ordem social, estética e até mesmo psicanalíticas, há efeitos políticos e jurídicos nesses processos que deveriam interessar aos/às juristas, notadamente questões de justiça ligadas à representação mediática e à maneira pela qual o direito considera atualmente os chamados "direitos morais" (direito ao nome, direito à imagem, direito ao respeito à vida privada, etc. $)^{16}$.

Como observa Brighenti ${ }^{17}$, o espaço público não é apenas o espaço de acesso comum que possibilita a discussão racional, mas é também o espaço de "afecções", de impressões afetivas que conduzem à ações irrefletidas. No domínio da afetividade

16 Cf. HEINICH, Nathalie. De la visibilité. Excellence et singularité en régime médiatique. Paris: Gallimard, 2012.

17 BRIGHENTI, Andrea. La démocratie a l'heure des visibilités hiérarchisées. In: nunca é possível excluir os efeitos afetivos ambivalentes ou incontroláveis advindos com a visibilidade de um grupo. Nesse sentido, a visibilidade reivindicada como direito é sempre uma via de mão de dupla, podendo se transformar em espetáculo, o que enseja distorções no processo de reconhecimento.

Por outro lado, prossegue Brighenti, é importante reconhecer que a visibilidade não depende apenas daquele/a que vê, mas igualmente daquele/a que é visto/a. Por isso, para alguns grupos sociais a visibilidade é ameaçadora. Por exemplo, moradores/as de rua no Brasil, sempre expostos/as à violência, percebem que a invisibilidade é uma estratégia de sobrevivência. Estar visível em uma rua de uma grande metrópole durante a madrugada pode representar a morte.

\section{Visibilidade, vigilância e controle}

Nicholas Mirzoeff tem procurado descrever a visibilidade como um dispositivo de poder.

FABRE, Thierry. La cité en danger. Dictature, transparence et démocratie. Rencontres d'Averroès 19, Marseille: Éditions Parenthèses, 2013. 
Questionando a celebração acrítica da expansão do visual e da proliferação de imagens no mundo contemporâneo, Mizoeff entende que a visibilidade tem a ver primeiramente com a vigilância, isto é, com as formas de disciplinar, normalizar e ordenar a visão.

Do ponto de vista de sua genealogia, entende Mirzoeff, a visibilidade se relaciona com a plantação escravista e o exército moderno. Nos dois casos a ideia é a mesma: trata-se da visibilidade como forma de conhecimento absoluto do "outro", forma assimétrica de visualização na qual não há reciprocidade do olhar. Contra essa articulação entre visibilidade e poder, Mizoeff reivindica um "direito de olhar", isto é, uma "contra-visualidade" que consistiria não apenas em ver as imagens de modo distinto, mas principalmente em criar estratégias de desarticulação do sistema hegemônico de visualização. A "contra-visualidade" estabeleceria um olhar mais igualitário, relacional e recíproco ${ }^{18}$.

18 Cf. MIRZOEFF, Nicholas. The Right to Look: A Counterhistory of Visuality. Durham, NC: Duke University Press Books, 2011.
De todo modo, convém ter clareza de que ser reconhecido/a pelo "outro" pode significar também ser observado/a por ele. A injunção da visibilidade na sociedade contemporânea está, de facto, ligada à vigilância e ao controle das pessoas, especialmente de determinados grupos de indivíduos ${ }^{19}$. No entender de Brighenti, está em curso na contemporaneidade uma reordenação dos regimes de visibilidade que amplia consideravelmente a margem do visível (e.g., satélites, tecnologias de geolocalização e de visualização miniaturizadas, microscópios, etc.), ao mesmo tempo em que expande o monitoramento e a vigilância nos espaços públicos e privados, algo que importa em demasia ao direito.

Já Foucault (1987) havia alertado para essa onipresença dos dispositivos de controle e registro na sociedade contemporânea. Os estudos sobre visibilidade, entretanto, vão mais adiante e acrescentam um aspecto não previsto por Foucault. O sociólogo do direito Thomas Mathiesen, por

19 Sobre esse aspecto veja-se também MATTELART, Armand et Vitalis, André. Le profilage des populations. Paris: La découverte, 2014. 
exemplo, acrescenta ao modelo foucaultiano do panóptico (i.e., a vigilância que poucos exerceriam sobre muitos na sociedade disciplinar) o modelo do sinóptico como novo mecanismo de poder. Para Mathiesen, embora em diversas situações contemporâneas muitos indivíduos continuem a observar outros poucos, as novas tecnologias de informação e comunicação também permitem que muitos vigiem poucos, como acontece na televisão e na web.

$\mathrm{Na}$ sociedade atual, entende Mathiesen, a vigilância e o registro se tornaram tão banais quanto desejáveis, incidindo sobre muitos objetos diferentes: dados, meta-dados, perfis, estilos de vida, etc. O fenômeno global da observação ensejado pelo ciberespaço "democratiza" e inverte o controle: além da multiplicidade de meios de vigilância (e.g., câmeras, chips, drones, reconhecimento facial biométrico, etc.), as pessoas observadas são agora também observadoras recíprocas ávidas em verem e serem vistas. O panóptico e o sinóptico

20 Cf. GONZALO ABRIL, Cultura visual y espacio público-político. Cuadernos de Información y Comunicación 2010, vol. 15 21- operam, portanto, em conjunto nos dias atuais.

A possibilidade de uma ambivalência no uso das tecnologias vigilantes recebe o nome de sousveillance (Mann, 2004). Este neologismo remete à palavra francesa "surveillance" (que designa vigilância) e inclui o prefixo "sous", que nomeia o que está por baixo (sob). A "sousveillance" seria, deste modo, uma contra-vigilância feita, por exemplo, a partir de aparelhos portáteis, e que reduziria as disparidades entre quem controla e quem é controlado/a.

\section{Visibilidade mediática e democracia representativa}

Como observa Gonzalo Abril Curto, não existe ordem política que não tenha sustentado e expressado um regime de visibilidade, administrando o olhar e definindo o que pode ser visto e o que deve permanecer invisível ${ }^{20}$. $\mathrm{O}$ poder, em qualquer sociedade, requer uma "mise en scène", uma teatralização, conforme já havia assinalado Georges Balandier ${ }^{21}$. A

\footnotetext{
36.

21 Cf. BALANDIER, Georges. Pouvoir sur scène. Paris: PUF, 1980.
} 
novidade dos dias de hoje está no fato de que a atividade política passou a se desenvolver na esfera de uma visibilidade pública cada vez mais controlada pelos meios de comunicação social, operando a partir de uma lógica publicitária. A política passou a funcionar de modo semelhante aos produtos da indústria cultural, buscando captar a atenção do público no palco mediático, através de jogos e lutas simbólicas.

Por isso, o espetáculo, no sentido atribuído à expressão por Guy Debord, passou a ser outro aspecto da visibilidade, essencial para a manutenção do poder disciplinar na sociedade contemporânea. $\mathrm{O}$ espetáculo, no entender de Debord, tem dois sentidos que estão articulados, indicando tanto a centralidade da mercadoria no capitalismo avançado, quanto a transformação do real em imagens, isto é, a autonomização da representação frente à realidade: "O espetáculo é o momento em que a mercadoria ocupou totalmente a vida social. Não apenas a relação com a mercadoria é visível, mas não se

22 Cf. DEBORD, GUY. A Sociedade do Espetáculo. Rio de Janeiro: Contraponto, 1997. consegue ver nada além dela: o mundo que se vê é o seu mundo",22.

Ora, quais é que seriam as implicações decorrentes dessa espetacularização da política nos processos de tomada de decisões? Se, como diz Jacques Rancière ${ }^{23}$, a política ocupa-se "do que se vê e do que se pode dizer sobre o que é visto", de que forma é que os modos de ver e ser visto/a interferiram nos processos decisórios? É preciso primeiramente entender a própria transformação do espaço público como âmbito que organiza a experiência social no mundo contemporâneo. O desenvolvimento de dispositivos tecnológicos de mediação ensejou uma nova forma de comunicação que além de ter tornado a esfera pública muito mais complexa em termo de variedade, também a fez funcionar a partir de uma grande heterogeneidade de situações de interação, ampliando as formas tradicionais de visibilidade. No entender de John B. Thompson, esse novo cenário afetaria radicalmente a

23 Cf. RANCIÈRE, Jacques. Le partage $d u$ sensible. Esthétique et politique. Paris: La Fabrique, 2000. 
democracia em pelo menos dois grandes aspectos ${ }^{24}$.

Em seu célebre livro sobre as promessas não cumpridas da democracia, Bobbio afirma que esta nasceu com o propósito de fazer desaparecer o poder invisível, colocando em seu lugar um poder transparente, cujas decisões seriam sempre visíveis e controláveis por todos/as. Não obstante a crescente exigência contemporânea de exercício público do poder, que se dá por meio de uma série de conceitos e práticas (e.g., transparência, prestação de contas, acesso à informação, etc.), a imagem fortemente confiante da visibilidade na democracia vem sendo abalada pela forma como a visibilidade é mediatizada pelos meios eletrônicos. No entender de Thompson, esses meios - que não são apenas canais de transmissão da informação, mas são também elementos geradores de novas formas de ação e de interação - ensejam uma maior capacidade de controle pelos/as cidadãos/ãs, ao mesmo tempo em que produzem novas fragilidades sociais e demandas políticas

${ }^{24}$ THOMPSON, J. B. A mídia e a modernidade: uma teoria social da mídia. 2a ed. Petrópolis: Vozes, 1999. incompatíveis, tais como a exigência de administrações totalmente abertas e a existência líderes fortes e confiáveis.

Brighenti também tem procurado destacar outras distorções provocadas pela injunção das novas formas de visibilidade na democracia representativa contemporânea, em especial o retorno da "multidão". No entender de Brighenti, o grande debate sobre a multidão no século XIX representou uma tentativa de entender a nova situação social, espacial e material advinda com o desenvolvimento urbano das grandes metrópoles, o que produziu um conjunto de saberes sobre a multidão. Hoje, é preciso igualmente fazer frente a uma nova multidão que se configura antes de tudo como uma multidão de dados ensejada pelas novas tecnologias da informação e da comunicação. A nova multidão, entende o autor italiano, é pós-urbana e pós-humana: posts, comments, likes, tweets, feeds, etc. Porém, tal como ocorria com a multidão do século XIX, essa profusão caótica de dados é a constatação de um plenum social 
confuso e de uma visibilidade fortemente hierarquizada ${ }^{25}$.

Mas é preciso também reconhecer os novos arranjos e as possibilidades da esfera pública da contemporaneidade. Mesmo não atendendo a todas as exigências para se falar de uma efetiva discussão pública, as novas tecnologias virtuais possuem uma capacidade enorme de conectar indivíduos em redes que ultrapassam as limitações tradicionais de espaço e de tempo impostas às discussões off-line. Por outro lado, ao contrário dos meios de comunicação da época do apogeu da televisão, os novos meios virtuais possibilitam uma comunicação direta da informação política, sem intermediários/as, proporcionando que o público venha em tese a exercer um papel ativo e não o papel de simples apreciador do jogo político.

\section{Considerações finais}

O propósito deste breve trabalho, de traço fortemente exploratório, foi tentar destacar alguns aspectos do tema da visibilidade

25 BRIGHENTI, Andrea. Le nuove politiche di visibilità in rete. Cosmopolis. Revista di passíveis de serem explorados por uma reflexão teórica sobre o direito. Os estudos sobre cultura visual, ainda que incipientes no campo do direito, podem configurar uma estimulante abordagem do tema do reconhecimento social e jurídico de grupos minoritários, e oferecem perspectivas revigorantes sobre os temas da representação, segurança e democracia.

\section{Referências Bibliográficas}

Abril, Gonzalo. (2013) Cultura visual, de la semiótica a la política. Madrid: Plaza y Valdés.

\begin{abstract}
Abril, Gonzalo. (2010). Cultura visual y espacio público-político. Cuadernos de Información y Comunicación, vol. 15 21-36.
\end{abstract}

Aubert, Nicole e Harouche, Claudine. (2011). Tyrannies de la visibilité. Être visible pour exister? Paris: ERES.

Balandier, Georges. (1980). Pouvoir sur scène. Paris: PUF. 
Benjamin, Walter. (2013). L'EEuvre d'art à l'époque de sa reproductibilité technique. Paris, Payot.

Brighenti, Andrea. (2007). "Visibility: A Category for the Social Sciences", Current Sociology, n. 55, p. 323-342,

Brighenti, Andrea. (2009). Pour une territoriologie du droit, In: Forest, Patrick (Dir.) Géographie du droit. Épistémologies, développements et perspectives. Québec: Presses de l’Université Laval, pp. 239-260.

Brighenti, Andrea. (2011). Le nuove politiche di visibilità in rete. Cosmopolis. Revista di filosofia e teoria politica, $\mathrm{VI} / 2$.

Brighenti, Andrea. (2013). La démocratie a l'heure des visibilités hiérarchisées. In: FABRE, Thierry. $L a$ cité en danger. Dictature, transparence et démocratie. Rencontres d'Averroès 19, Marseille: Éditions Parenthèses.

Crary, Jonathan. (199). Techniques of Observer. On vision and modernity in the XIX century. Massachusetts, MIT Press.
Debord, Guy. (1997). A sociedade do Espetáculo. Rio de Janeiro: Contraponto.

Fiserová, Michaela. (2013) Partager le visible. Repenser Foucault. Paris: L'Harmattan.

Foucault, Michel. (1987). Vigiar $e$ punir: nascimento da prisão. Petrópolis: Vozes.

Haroche, Claudine. (2008) A condição sensível. Rio de Janeiro: Contracapa.

Heinich, Nathalie. (2012). De la visibilité. Excellence et singularité em régime médiatique. Paris: Gallimard.

Honneth, A. (2005). La lutte pour la reconnaissance. Paris: Éditions le Cerf.

Honneth, A. (2004). L'invisibilité: sur l'épistémologie de la reconnaissance». Réseaux, vol. 23, n. 129-130, p. 41-57. Jay, Martin (1996). Introduction: Vision in context: reflections and refractions. In : BRENNAN, T. ; JAY, M. (Ed.) Vision in context: historical and contemporary perspectives on 
sight. New York/London: Routledge,

1996.

Mattelart, Armand et Vitalis, André.

(2014) Le profilage des populations.

Paris: La découverte.

Mirzoeff, Nicholas. (1998) Visual

Culture Reader. London: Routledge.

Mirzoeff, Nicholas. (2011). The Right

to Look: A Counterhistory of Visuality.

Durham, NC: Duke University Press

Books.

Ranciere, Jacques. (2000). Le partage

du sensible. Paris: La Fabrique.

Rawls, John. (1999). A Theory of justice. Harvard Universty Press.

Sauvageot, Anne. (1994). Vois et Savoirs. Esquisse d'une sociologie du regard, PUF.

Thompson, J. B. (1999) A mídia e a modernidade: uma teoria social da mídia. 2a ed. Petrópolis: Vozes.

Voirol, Olivier. (2005) Visibilité et invisibilité: une introduction. Reseaux, $\mathrm{n}^{\circ} 129-130$, p. 9-36. 